\title{
Electron Microscopy in the Analysis of Renal Biopsies: Always the Last Step?
}

\author{
David N. Howell, MD, PhD
}

\section{Department of Pathology, Duke University and Veterans Affairs Medical Centers, Durham, NC, USA}

Electron microscopy (EM) plays a key role in the evaluation of renal biopsies. It is typically regarded as the final step in a diagnostic sequence that begins with light microscopy (LM) and immunofluorescence microscopy (IF). Since LM and IF frequently provide considerable information about a biopsy, consideration is occasionally given to dispensing with the terminal EM examination. In many instances, this would lead to loss of critical information, including the precise location, consistency, and substructure of immune deposits detected by LM/IF as well as a wide array of features that are invisible by optical microscopic methods [1]. It has been estimated that EM provides important diagnostic information for $50 \%$ of renal biopsies, even when it brings up the rear in the analytic process [2].

In a surprising number of cases, however, EM is actually the midpoint of a complex diagnostic process rather than the terminus, serving both as a source of information and a guide to subsequent investigative steps. This is particularly true if the disease process involved is rare and/or clinically unexpected. With the ultrastructural findings as a clue, additional valuable information can often be gleaned by a thorough review of the clinical record (occasionally extended by reexamination of the patient) and careful reevaluation of LM, IF, and EM studies already performed. Frequent next steps include special studies performed on the tissue samples used for LM (histochemistry, immunohistochemistry [IHC], in situ hybridization), IF (immunofluorescent staining for $\alpha$ chains of type IV collagen or IgG subclasses), and EM (morphometric analysis, immunoelectron microscopy). In selected cases, follow-up clinical laboratory testing (molecular diagnostic tests for mutations, enzyme assays) or additional biopsy (e.g., when EM provides evidence of a possible occult malignancy) may be indicated.

Lymphomas occasionally produce clonal immunoglobulins that lodge in the renal glomerulus. Examples include deposits of IgM, often in the form of large, intraluminal collections on non-structured material, frequently associated with Waldenström macroglobulinemia/lymphoplasmacytic lymphoma (LPL) and deposits of IgG in the form of microtubules, referred to as immunotactoids, often associated with small lymphocytic lymphoma/chronic lymphocytic leukemia (SLL/CLL). Both of these lymphomas can easily be mistaken for benign inflammatory infiltrates if they are not suspected. Though the lymphoma itself is often confined to lymphoid tissue, renal lymphomatous infiltrates are occasionally present. An example of immunotactoid glomerulopathy is provided in Fig. 1A; on review, an innocuous-appearing lymphoid infiltrate (Fig. 1B) was shown to be an occult SLL/CLL by IHC.

A wide variety of non-immunoglobulin deposits, many of which require further diagnostic investigation, can also be detected by EM. More than two dozen forms of non-AL amyloidosis have been described; EM is a sensitive method of detecting the characteristic amyloid fibrils, but does not allow distinction of the constituent proteins. Testing by other techniques, including IHC and laser microdissection/mass spectrometry, is required to ascertain the type of amyloid present. Several heritable enzyme deficiency disorders, including Fabry disease and lecithin-cholesterol acyltransferase (LCAT) deficiency, can also be detected by the presence of characteristic glomerular deposits of enzyme substrate. Confirmation of the diagnosis is often sought using assays for the missing enzyme ( $\alpha$-galactosidase A for Fabry disease) or its expected product (cholesterol esters for LCAT deficiency) or genomic mutation analysis. 
The initial diagnosis of hereditary nephritis due to mutations in the genes encoding the $\alpha 3$, $\alpha 4$, and $\alpha 5$ chains of type IV collagen is often accomplished by EM, particularly in patients without a family history of the disorder; individuals with non-syndromic or atypical presentations; and female carriers of X-linked Alport syndrome, for whom random inactivation of X chromosomes during embryogenesis leads to lack of expression of functional collagen proteins in a mosaic pattern. Ultrastructural detection of the characteristic thinning, thickening, and lamellation of glomerular capillary loop basement membranes can be confirmed by immunofluorescent staining for the relevant collagen chains or by mutation analysis, though the latter is quite expensive. Fig. $1 \mathrm{C}$ shows a mosaic pattern of glomerular basement membrane abnormality detected by EM in an unsuspected carrier of X-linked Alport syndrome; Fig. 1D is a confirmatory immunofluorescent stain for the $\alpha 5$ chain of type IV collagen showing similar mosaic expression of the protein in a glomerulus.

The examples above hopefully provide convincing evidence that EM can be both a definitive endpoint and a crucial interior step in the analysis of renal biopsies. In special circumstances, EM actually provides the initial information in a diagnostic chain, as when the detection of polyomaviruses in the urine of renal transplant recipients by negative staining prompts a biopsy for diagnosis of polyomavirus tubulointerstitial nephritis. Most nephropathologists have also encountered occasional biopsies in which diagnostic tissue was only present in the sample apportioned for EM; even a small fragment of a glomerulus is sometimes sufficient to render an ultrastructural diagnosis [1]. The reader is encouraged to keep an open mind about application of EM at any phase in the analysis of renal biopsies.

\section{References:}

[1] EN Pavlisko and DN Howell, Ultrastruct. Pathol. 37 (2013), p. 1-8.

[2] M Haas, J. Am. Soc. Nephrol. 8 (1997), p. 70-76.

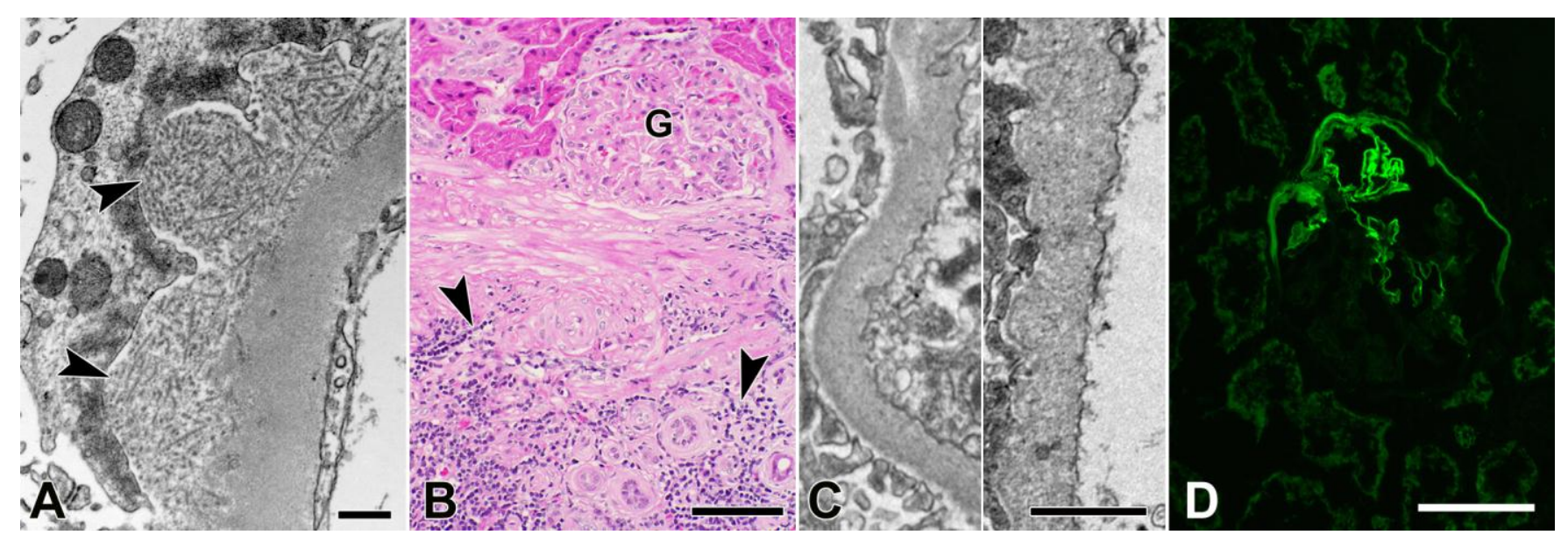

Figure 1. (A) Electron micrograph showing immunotactoids (arrowheads) and (B) light micrograph showing glomerulus $(\mathrm{G})$ and lymphomatous infiltrate (arrowheads) in patient with immunotactoid glomerulopathy and SLL/CLL. (Bar in $\mathrm{A}=500 \mathrm{~nm}$; bar in $\mathrm{B}=100 \mu \mathrm{\eta}$.) (C) Electron micrograph showing normal (left) and lamellated (right) basement membranes and (D) immunofluorescence micrograph showing mosaic staining for $\alpha 5$ chain of type IV collagen (staining restricted to upper half of glomerulus) in carrier of $\mathrm{X}$-linked Alport syndrome. (Bar in $\mathrm{C}=1 \mu \mathrm{m}$; bar in $\mathrm{D}=100 \mu \mathrm{m}$.) 